\title{
Constraints on stellar evolution from white dwarf asteroseismology
}

\author{
Agnès Bischoff-Kim
}

Penn State Worthington Scranton email: axk55@psu.edu

\begin{abstract}
High mass and low mass stars follow a similar evolution until the inert core phase that follows the end of the core helium burning stage. In particular, one common phase of stellar evolution is the alpha capture reaction that turns carbon into oxygen in the core. We can obtain constraints on this reaction rate by studying the remnants of low mass stars, as this is the ultimate reaction that occurs in their core. We also present results that allow us to test the time dependent calculations of diffusion in dense interiors.
\end{abstract}

Keywords. dense matter, diffusion, nuclear reactions, stars: AGB and post-AGB, stars: interiors, stars: oscillations, white dwarfs

\section{Introduction}

A small subset of white dwarfs pulsate in non-radial, g-mode oscillations. These stars have been used to study a number of fundamental physical processes (e.g Bischoff-Kim et al. 2008; Bischoff-Kim 2008; Córsico et al. 2013), including the emission of weakly interacting particles. Pulsating white dwarfs are useful because we have a good set of observed frequencies for a dozen of them and they are relatively simple to model (neglible nuclear burning, no extended atmospheres, non-contracting, well determined equations of states...). With white dwarf asteroseismology, we can test stellar evolution calculations. We focus here on two processes: i) the ${ }^{12} \mathrm{C}(\alpha, \gamma){ }^{16} \mathrm{O}$ nuclear reaction rate (section 2) and ii) the diffusion of elements over time in stellar interiors (section 3).

In white dwarf asteroseismology, we parameterize the core chemical profiles and allow them to take the shape required to have the models' frequencies match the observed frequencies. This is in contrast with stellar evolution based asteroseismology, where the chemical profiles are calculated based on nuclear reaction rates, diffusion, convection and other other relevant physical processes. While perhaps less physical, such modelling ties internal structure more directly to the observed frequencies. Results of white dwarf asteroseismology can then be confronted with what stellar evolution calculations yield. For more details on the models and methods used in this study, we refer the reader to Bischoff-Kim \& Østensen (2011).

\section{The ${ }^{12} \mathrm{C}(\alpha, \gamma){ }^{16} \mathrm{O}$ rate}

Two white dwarfs for which we have pulsation spectrums of high enough quality for detailed asteroseismic studies are GD 358 and CBS 114, both helium atmosphere white dwarfs. Metcalfe (2003) determined what the rates of the ${ }^{12} \mathrm{C}(\alpha, \gamma){ }^{16} \mathrm{O}$ nuclear reaction needed to be in order to produce the carbon and oxygen abundance profiles infered from the asteroseismic study of GD 358 and CBS 114. For both stars, the rates found were consistent with the NACRE nuclear reaction rates (Angulo et al. 1999). 


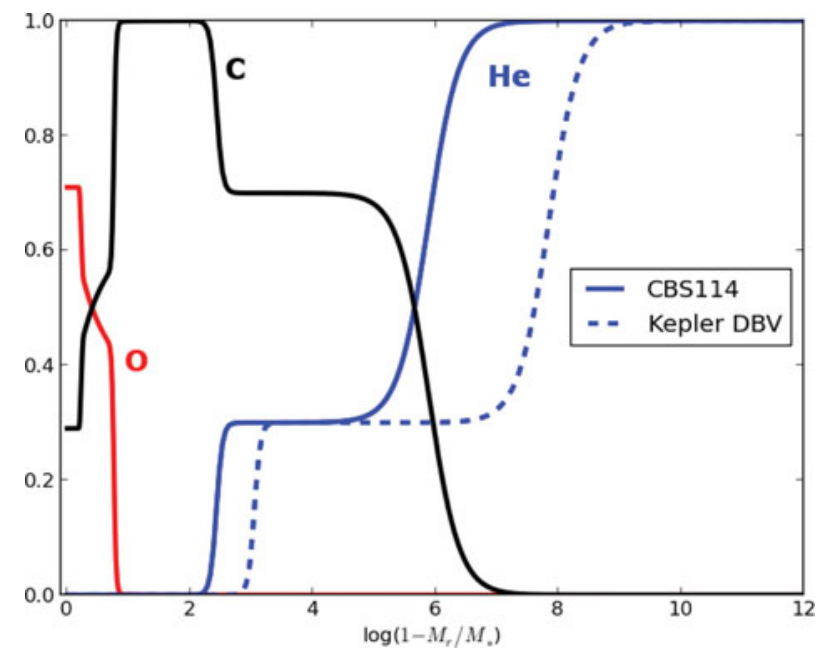

Figure 1. Composition profiles for helium atmosphere pulsating white dwarfs. Each profile is the result of asteroseismic fits. The basic shape of the profiles was borrowed from results of time-dependent diffusion calculations (Dehner \& Kawaler 1995) and parameterized. The carbon and oxygen profiles are for CBS 114. For clarity, the carbon and oxygen profiles for the Kepler DBV are not shown.

\section{Diffusion of helium}

Another example of the use of white dwarf asteroseismology to test stellar evolution calculations is the diffusion of helium. For helium atmosphere white dwarfs, helium is the lightest element present. Time dependent calculations predict that over time, helium floats up to the surface (e.g. Dehner \& Kawaler 1995). The asteroseismic study of white dwarfs in different stages in their evolution qualitatively confirm this. Asteroseismic studies of CBS 114 (Metcalfe et al. 2005) and KIC 8626021, a helium atmosphere white dwarf (DBV) found in the original Kepler field, show that the helium layer in CBS 114 is thicker than the helium layer in the Kepler DBV. The Kepler DBV is hotter than CBS 114, meaning it is younger. This result is shown in Fig. 1.

\section{Summary and Future Work}

White dwarfs hold fossilized in their core, the results of nuclear synthesis on the AGB. Some white dwarfs pulsate and we can probe their interior structure, allowing us to test these nuclear reaction rates. We also observe a trend in the thicknesses of their helium envelopes, consistent with the outward diffusion of helium as white dwarfs cool. This trend needs to be quantitatively checked against time dependent diffusion calculations.

\section{References}

Angulo, C., Arnould, M., Rayet, M., et al. 1999, Nuclear Physics A 656, 3

Bischoff-Kim, A. 2008, Communications in Asteroseismology 154, 16

Bischoff-Kim, A., Montgomery, M. H., \& Winget, D. E. 2008, ApJ 675, 1512

Bischoff-Kim, A. \& Østensen, R. H. 2011, ApJl 742, L16

Córsico, A. H., Althaus, L. G., García-Berro, E., \& Romero, A. D. 2013, Journal of Cosmology and Astroparticle Physics 6, 32

Dehner, B. T. \& Kawaler, S. D. 1995, ApJl 445, L141

Metcalfe, T. S. 2003, ApJl 587, L43

Metcalfe, T. S., Nather, R. E., Watson, T. K., et al. 2005, A\&3 A 435, 649 